\title{
Examining Interactions in Social Network Sites through the Lense of Social Capital
}

\author{
Stephen. K. Musembwa \\ Nova Southeastern University \\ sm1447@mynsu.nova.edu
}

\author{
Souren Paul \\ Northern Kentucky University \\ souren.paul@gmail.com
}

\begin{abstract}
Rapid growth of the Internet has led to the proliferation of technology, including the use of social network sites (SNS). Social network sites facilitate communications between online users with shared interests and enable users to share content seamlessly. Accordingly, the rapid growth of social network site usage necessitates analysis of factors affecting usage of SNS and the creation of social networks on the social network sites. A research model that focuses on social capital in SNS and the development of community in SNS is proposed in this paper. The model suggests how the configuration of SNS and the diversity of SNS users influence different relational facets of social capital such as trust, reciprocity and identification needs in SNS and the sense of community in SNS. We conducted a web-based survey to collect the data to test our hypotheses. We find that SNS users interaction needs and trust in interaction have positive relationships with reciprocity in SNS interactions. We also find that the development of the sense of community promotes effective communication in SNS.
\end{abstract}

\section{Introduction}

Social network sites (SNS) are becoming popular and we find tremendous growth in the formation of groups and communities in these sites. These groups or communities have common interests and/or common sources of relationships. While some of these groups/communities do not grow over time, there are many groups/communities that become popular and grow very rapidly [39]. Some common examples are book clubs, academic researchers, software developers, cultural groups, business executives and more. Given that SNS in general and SNS communities in particular are becoming very popular, their rapid growth inspire us to conduct a study to understand the factors that foster a continuing sense of communities in SNS. In addition, we want to examine if the development of the sense of community facilitates the effectiveness of communication in SNS.

Social network sites offer settings where users can share ideas, texts, photographs, videos and more with individuals of the same backgrounds and interests. Typically, social network sites avail privacy settings to allow users to choose 'friends' that can view or add content to their personal pages on the networks [35]. Accordingly, privacy control settings are crucial, because, if left un-activated, a user's personal web page is available to the online universe to make changes [35].

Additionally, the ubiquity of wireless telephony devices has led to the rapid growth of mobile applications related to social network sites [25]. This extraordinary growth of wireless devices leads to increased usage of social networks. Online users from different regions and countries are increasingly forging relationships in internet-related social networks and communities. Participation in social networks sites is regional, global and can be between individuals from diverse social and cultural backgrounds. Accordingly, cultural belonging among users may affect aspects of group interaction including trust and reciprocity [24].

Although, social networks sites attract new entrants at a rapid rate, there are numerous drawbacks related to SNS usage. Occasionally social network users freely divulge personal information leading to the potential of exploitation of personal information by devious SNS users [35]. This poses threat to the having continued interactions via SNS.

Prior studies on SNS have examined the relationships between self-esteem and social capital [36]; between cultural differences and the motivations for using SNS [16]; between certain kinds of Facebook use and formation of social capital [8]; asymmetric communication in Facebook and bridging social capital i.e. access to new information through a diverse set of acquaintances [4]; directed communication and the feelings of bonding social capital i.e. emotional support from close friends [5]. Thus, prior studies on SNS indicate that social capital is an important construct. 
However, there is no comprehensive framework on various facets of social capital in SNS and the relationship between social capital and the development of community in SNS. We attempt to address this gap by building a theoretical model that links the configuration of SNS and various sources of diversity of SNS users with different relational facets of social capital (i.e. trust, reciprocity, and identification needs) in SNS and the sense of community in SNS. We focus on the following research questions in this paper:

1. Does diversity (cultural and native language) affects trust and reciprocity in SNS interactions?

2. Does the configuration of social networks affect trust and reciprocity in SNS interactions?

3. Does identification need affect reciprocity in SNS interactions?

4. Do reciprocity and trust in SNS interaction reinforce affect the development of the sense of community in SNS?

5. Does the sense of community affect the effectiveness of communication in SNS?

The theoretical model proposed in this paper is presented in the figure 1 .

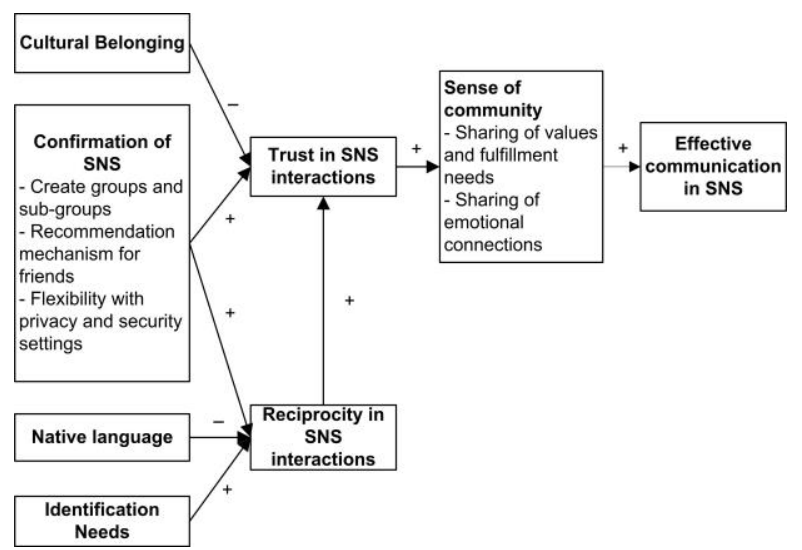

Figure 1: Research Model

\section{Literature Review}

The theoretical model presented in figure 1, has its roots in prior studies on social capital [31]; [29]. Social capital is "the ability of actors to secure benefits by virtue of membership in social networks or other social structures" [10]. Nahapiet and Ghosal [29] suggest three dimensions of social capital, which are the structural, the relational, and the cognitive dimensions. Structural dimensions are the "properties of the social system and the network of relations as a whole" [29]. The focus is on "impersonal configuration of linkages." The relational dimension refers to the "personal relationships" that people develop in a social system. The cognitive dimension refers to "shared representations, interpretations, and systems of meaning" [29]. The structural dimension includes the facets like, network ties and network configuration while the relational dimension is characterized by trust, norms, obligation, and identification needs of the actors in a social system. Shared language, codes, and narratives constitute the cognitive dimension of social capital [29]. Nahapiet and Ghoshal [29] focused only on the group level factors of social capital. However, individual level interactions contribute to form a larger network. The individual level interactions are the basic source to determine how an individual behaves in relation to another [40]. Furthermore, in study [29], the electronic networks are considered in relation to the organizational advantages, without evaluating these factors in terms of large communities on SNS.

We consider the aforementioned dimensions of social capital to extend the concept interactions in SNS. We focus on the configuration of SNS, the relational issues (trust, reciprocity in SNS interaction, and identification needs), and the existence of shared language and narratives as the primary dimensions of social capital in SNS-based interactions. We link these dimensions to the development of the sense of community. We consider some interrelationships among these facets of SNS social capital and include the construct diversity, which is important for SNS. We present the literature review and build the theory in the subsequent sub-sections of this paper.

\subsection{Cultural Belonging}

The six initial culture dimensions of the GLOBE program originated from Hofstede's cultural model [13]. GLOBE and other frameworks updated Hofstede's cultural dimensions based on the evolving global cultures and economic environments [38]. GLOBE underscores culture as shared values amongst individuals with common experiences [14]. Users that join social network sites may initially seek out SNS familiar members with similar cultures or values [9]. Accordingly, the author proposed that cultural belonging could have an effect on trust, because, trust is most likely higher in social networks where members are culturally similar and less where members are culturally diverse [28].

Alternatively, there are studies about the effect of motivation of SNS usage based on cultural difference [15]. Researchers posit that it is difficult to utilize Hofstede's theory into current studies, because of the perpetual dynamic change in cultural dimension, in that past culture was characterized as immobile and this does not reflect the current phenomenon of rapid cultural 
change [17]. In addition, the expansion of the Internet provides opportunities for users to transact on a global scale, whereby the influences of the Internet are affecting the homogenization of cultural components in online environments such as SNS [32]. It is quite possible that members from divergent backgrounds can eventually develop trusting relationships with others, once all parties become familiar with one another to form social networks in SNS.

\subsection{Native Language Variation}

Native language variation refers to the differences in linguistic influences mainly tied to ethnicity. Language variation entails the different languages spoken and the various people who speak the languages [33]. Language has a critical purpose in social relations, because it is the channel used by people to confer and share information, shared language facilitates interaction between people as they that interrelate. When people share a common language, it increases their ability to gain access to people that speak that language [29]. On the other hand, when language and social codes differ, people could stay apart, and this could restrict their access to each other's information [28].

Melitz and Touba [27] found that linguistic factors have an impact on social interactions in that individuals communicating in a common language gravitate towards each other. Interestingly, the researchers in the same study found that ease of communication is more significant than ethnicity and trust in social interactions [27]. Individuals that speak the same language are more apt to easily communicate, form societal bonds and reciprocate each other's actions.

\subsection{Configuration of SNS}

Configuration of SNS refers to the architectural and technical design of the SNS that typically, entails availing privacy settings to allow users to choose 'friends' that can view or add content to their personal pages on the networks [35]. Social network sites as online forums enable users to create profiles, connect and share information with other users that have similar interests [3]. Typically, SNS users set their privacy settings prudently, favoring users that they trust or have reciprocal relationships with. Accordingly, configuration of the SNS is a key component of the SNS experience.

Once users create the networks, other users in their online cliques can show approval by 'faving' or 'liking' uploaded content. In addition, users can expand lists of their 'favorites' contacts via inter-connections between the web of online social networks [20]. The 'faving' element is a key component of content sharing as it propagates content and facilitates user participation [20]. Favoring or "faving" facilitates approval by content consumers who are typically users in a given network. In order to show their gratitude and stimulate further communications, at times users reciprocate by sharing their own content, as a token of their appreciation [20].

Privacy control settings when activated, enable a user to share the personal web page to a selected audience in the online universe [35]. In order to control the unmitigated access to personal pages, SNS users typically set their privacy settings prudently, favoring users that they trust or have reciprocal relationships with. Accordingly, the configuration of SNS could have an influence on trust and reciprocity among SNS users.

\subsection{Identification Needs}

Identification is as a mode of social influence that refers to the self-consciousness of one's affiliation to a group, as well as the emotional importance of this affiliation [37]. Identification occurs when a person consents to influence of a group because they want to ascertain a relationship with that group. Identification is also defined as a process where people perceive themselves as one with another person or group of people, because of their membership in the group or as a reference to the group, through the group's operations [29]. Group identification can increase the perceived opportunities for exchange and enhance the actual frequency of collaboration between interacting parties [22]. Accordingly, identification acts as a resource influencing both the anticipation of value to be attained through interaction and the motivation to exchange information by individuals and groups [29].

Consequently, the psychological status belonging to a community in an online social network can be stem from affective social identity, evaluative social identity and cognitive social identity [6]. Affective social identity is a sense of emotional connection with the community, evaluative social is an assessment of selfworth based on one's belonging to a specific group and cognitive social identity is a sense of awareness of an individual being part of a community [6]. We expect that these three components of social identity will influence a person's likelihood of use a social networking site. Essentially, if a user holds strong social identity toward an SNS their intention to use the SNS should increase. A stronger social identity leads to a stronger sense of belonging and higher likelihood of participating in an online SNS. 


\subsection{Trust in SNS interactions}

Trust is the inclination of a person to be receptive to and expect certain actions of another person, regardless of the ability of the trustor to monitor the actions other party [11]. Social network sites' members characteristically join sites if they are interested in the social online events or obtain a level of trustworthiness in the SNS. Trustworthiness in SNS depends on various factors, such as privacy and perceptions of trust of the SNS [7]. Accordingly, trust eventually grows in social network sites, if group members and users believe in the safety and privacy procedures implemented on the social network site [9]. Researchers have posited that the greater the trust levels in a social network site, the higher the likelihood of new users joining the site [9]. SNS users' willingness to disclose personal information and nurture new online relationships is influenced by perceptions of trust and privacy [7]. Trust is an essential component in the facilitating information exchange and providing valuable information in SNS. Existence of high levels of trust, typically translates to people being more willing to provide support to other SNS members [18].

\subsection{Reciprocity in SNS interactions}

Reciprocity is defined as a user's strategy to return favors received from others, in a manner comparable to the receiving method [20]. Reciprocity is a notion where people aid others, because others have assisted them in the past and they expect the same treatment in the future [19]. Researchers have found that reciprocity can lead to more trust, connectivity and cohesion within a group [1].

The concept of reciprocity includes content rating or faving and reciprocity actions discerned in Flickr and Twitter and more. Lee, Antoniadis and Salamatian [20] found that reciprocity is a fundamental aspect of the human psychology and online behavior. Lee, Antoniadis and Salamatian [20] argue that two SNS users could favor each other's photos because they like them. Alternatively, the faving deed could generate gratification for the receiver, who in turn could feel obligated to reciprocate. Additionally, Lee, Antoniadis and Salamatian [20] found in their research that faving reciprocity plays a significant role in social networks in that the more the outgoing favorites of a user, the more chances that the user will obtain favorites on their content.

Trust, social interaction and reciprocity are mutually reinforcing constructs, because trust facilitates knowledge sharing and fosters reciprocal actions. Accordingly, the ability to achieve reciprocity is crucial to building social networks in SNS.

\subsection{Sense of Community and Effective Communication in SNS}

Sense of community entails four separate dimensions: membership, influence, integration and shared emotional connection [26]. Membership refers to the consciousness of being part of a group; influence refers to the feeling that an individual is relevant to the group, and the group can influence its members; integration indicates that the wishes of members are met by the resources received through their affiliation to the group and shared emotional connection refers to the sense of shared history of a community [26].

Effective communication refers to articulateness or the ability of individuals to express popular and or unpopular dissenting opinions [23]. Effective communication within a SNS entails the fluency, eloquence and nature of expression between the members of the SNS. Effective communicators should be able to express popular and dissenting views coherently, without being offensive to other members of a SNS.

Community activities and their development require entities around which joint interests can be organized, which lead to sense of community [29]. A great deal of social capital is entrenched within networks of shared acquaintance and recognition, where users feel a sense of community [29]. Accordingly, since meaningful communication is an essential component of social interaction, development of sense of community facilitates effective communication in a SNS.

\section{Theory Development}

The theoretical model in this paper links the various relational facets of social capital in SNS and the sense of community in SNS. The model examines how the multiplicity of SNS users influence different relational facets of social capital such as trust, reciprocity and identification needs in SNS and the sense of community in SNS. The network-based theory of social capital identifies key aspects of patterns of social relations, where people with social ties participate in reciprocal interactions [23].

Although new members of SNS may eventually "friend' individuals with dissimilar cultures or values as initial trust maybe non-existent, users that join social network sites, typically seek out SNS familiar members that have similar cultures or values [10]. Generally, cultural belonging has an effect on trust, because, trust is most likely higher in social networks where members are culturally similar and less where members are culturally diverse. Accordingly, we hypothesized that: 
H1: Cultural belonging has a positive effect on trust in SNS interactions.

People with the same native language background will understand each other's messages easily and will feel comfortable responding to these messages. Accordingly, people that communicate in the same native language tend to gravitate towards each other. Conversely, users of a SNS that have different backgrounds in terms of native language may decipher messages from other SNS users differently and the exchange of messages between these divergent users may not be very smooth. Therefore, we hypothesized that:

H2: Native language variation has a negative effect on reciprocity in SNS interactions.

SNS users set their privacy settings favoring users that they trust or have reciprocal relationships with; SNS enable users to create profiles, connect and share information with other users that have similar interests. In addition, social network sites avail faving and privacy settings to allow users to choose 'friends' that can view or add content to their personal pages on the networks [35]. Because unmitigated access can lead to security and privacy violations, SNS users typically set their privacy settings favoring users that they trust or have reciprocal relationships with. Accordingly, the configuration of SNS could have an influence on trust and reciprocity among SNS users. Therefore, we hypothesized that:

H3a: Configuration of SNS has a positive effect on trust in SNS interactions

$H 3 b$ : Configuration of SNS has a positive effect on reciprocity in SNS interactions

Groups in SNS provide users with a sense of belonging, which enhances their identification needs with the social network in the SNS. A stronger social identity leads to a stronger sense of belonging and higher likelihood of participating in an online SNS. Accordingly, sense of belonging could potentially facilitate reciprocal actions in SNS interactions, as users in the same group would most like perform reciprocal actions. Therefore, we hypothesized that:

H4: Identification needs have a positive effect on reciprocity in SNS interactions.

Trust is a key component in the facilitation of information exchange in SNS. In addition, trust is both a precursor and result of effective collaboration. High levels of trust typically translate to people being more willing to provide support to other SNS members [23]. Accordingly, the greater the trust levels in a social network site, the higher the likelihood of new users joining the site. Therefore, we hypothesized that:

H5: Trust in SNS interactions has a positive effect on sense of community.
The ability to achieve reciprocity is crucial to building social networks in SNS. A network with numerous reciprocal linkages is likely to be more robust than one with fewer links of this nature. Prior research on games and economics suggest that reciprocity is a basic element of human behavior and it accounts for trust in anonymous counterparts [2]. Therefore, we hypothesized that:

H6: Trust in SNS interactions increases with increased reciprocity in SNS interactions.

Effective communication is an essential part of social exchange and meaningful communication is an essential component of social interaction. A great deal of social capital is entrenched within networks of shared acquaintance and recognition, where users feel a sense of community [29]. Accordingly, since meaningful communication is an essential component of social interaction, development of sense of community facilitates effective communication in a SNS. Therefore, we hypothesized that:

H7: Sense of community has a positive effect on effective communication in SNS.

\section{Research Method}

This section discuss our research method, subjects, identification of the variables and a description of the study.

\subsection{Research Design and Subjects}

A web-based survey was deemed as suitable method for this study. The target participants were current SNS users that are adults and reside in the United States. The participants were identified and targeted based on criteria configured in web-based survey and distributed via email. For the purposes of this study, the active users of SNS are targeted to evaluate the presented hypothesis. The survey was sent to the participants in the month of November of 2018. Respondents completed the survey in a period of two weeks. The subjects in the study were adults in the U.S. that use SNS. The survey instrument was created using a Webbased survey format. The survey was sent to 467 adults and there were 259 complete responses to the survey. Overall, the response rate was $55 \%$. Of the respondents with complete responses, 124 , or $47.9 \%$, were male, while 135 , or $52.1 \%$, were female. Over $70 \%$ of the respondents had been member of a SNS for over 3 years. The age groups varied with most respondents falling between 30-44. 


\subsection{Operationalization of Variables}

We calculated reliability of the instrument using Cronbach's Alpha coefficient for each if the variables in the study. An alpha of 0.831 was found for configuration of SNS, 0.897 for identification needs, 0.908 for trust, 0.851 for reciprocity, 0.937 for sense of community, and 0.894 for effective communication. This analysis established that all the items were reliable as the estimates for reliability for all constructs were above 0.8 , which exceeded the recommended threshold of 0.70 [10].

In order to validate the constructs, we conducted exploratory factor analysis using Varimax orthogonal rotation for the instrument. The researcher performed factor analysis with principal component analysis and varimax rotation as presented in Table 1. A Kaiser Meyer-Olkin test for constructs was run and the results were above 0.70 , signifying adequate sampling for factorability of the items. The factor analysis of four items representing sense of community loaded on a single factor and resulted in factor loadings ranging from 0.825 to 0.864 . The factor analysis of three items representing effective communication loaded on a single factor and resulted in factor loadings ranging from 0.809 to 0.849 . The factor analysis of four items representing identification needs loaded on a single factor and resulted in factor loadings ranging from 0.629 to 0.779 . The factor analysis of four items representing configuration of SNS loaded on a single factor and resulted in factor loadings ranging from 0.760 to 0.847 . The factor analysis of six items representing trust and reciprocity loaded on two factors and resulted in factor loadings ranging from 0.542 to 0.836 .

Discriminant validity is extent to which a construct is truly distinct from other constructs. Discriminant validity tests that constructs that should have no relationship do, in fact, not have any relationship [21]. We examined discriminant validity by analyzing the indicator items measuring configuration of SNS, identification needs, trust, reciprocity, sense of community, effective communication. We performed factor analysis with VARIMAX rotation involving a joint set of 22 indicator items that produced five different factors that we identified as "trust and reciprocal interactions" "sense of community", "effective communication" "identification needs" and "configuration of SNS". The results exhibit reasonable discriminant validity. The researcher noted the distinguishable constructs had items that load effectively on their respective constructs for identification needs, configuration of SNS and effective communication.

Table 1: Rotated component matrix ${ }^{1}$

\begin{tabular}{|l|c|c|c|c|c|}
\hline \multicolumn{5}{|c|}{ Component } \\
\cline { 2 - 6 } & 1 & 2 & 3 & 4 & 5 \\
\hline TR1 - Take advantage & .836 & & & & \\
\hline TR3 - Knowingly disrupt & .826 & & & & \\
\hline TR5 - Truthful dealing & .804 & & & & \\
\hline TR4 - Behave consistently & .773 & & & & \\
\hline TR2 - Keep promises & .765 & & & & \\
\hline RP2 - Reciprocity Group & .577 & & & & \\
\hline SC3 - Enrich knowledge & & .864 & & & \\
\hline SC1 - Successful functioning & & .836 & & & \\
\hline SC2 - Continue operation & & .828 & & & \\
\hline SC4 - Community growth & & .825 & & & \\
\hline RP1 - Reciprocity Myself & .542 & & & & \\
\hline EC3 - Time interacting & & & .849 & & \\
\hline EC1 - Frequent communication & & & .809 & & \\
\hline EC2 - Close relationships & & & & .779 & \\
\hline ID1 - Belonging & & & & .706 & \\
\hline ID2 - Closeness & & & & & \\
\hline ID3 - Positive feeling & & & & & .7629 \\
\hline ID4 - Proud member & & & & & \\
\hline CG4 - Privacy settings & & & & & \\
\hline CG2 - Create groups & & & & & \\
\hline CG3 - Hide friends & & & & \\
\hline CG5 - Privacy controls & & & & \\
\hline
\end{tabular}
${ }^{1}$ Extraction Method: Principal Component Analysis Rotation Method: Varimax with Kaiser (5 components extracted, and the rotation converged
in 6 iterations) Normalization 
The researcher noted that KMO Measure of Sampling Adequacy for all items were above 0.7 and Bartlett's test of Sphericity score were less than 0.05. In addition, the researcher noted that the factors loadings for all items after rotation, loaded significantly on their respective constructs and invariably loaded above 0.500 [9]. This meets the convergent validity requirement.

The survey was designed with questions related to each of the variables. The variables were measured based on attributes selected by the participants. The survey contained a total of 28 questions denoting the attributes presented to the participants and 7 questions related to demographics.
The survey was broken up to into five sections. The first section of the survey instrument addressed configuration of SNS sites, identification needs, trust is SNS interactions and reciprocity is SNS interactions and consisted of 16 items on a seven-point Likert scale. The second section of the survey instrument addressed sense of community and effective communication and consisted of 8 items on a seven-point Likert scale. The third section of the survey instrument addressed cultural belonging and native language variation and consisted of 4 items on a yes/no scale. The final section consisted of variables related to the demographics, including gender, age, education level, country of residence and years of SNS use.

Table 2. Regression results [Hypotheses Test 1-7]

\begin{tabular}{|c|c|c|c|c|c|c|c|c|}
\hline \multicolumn{4}{|l|}{$\begin{array}{l}\text { Dependent } \\
\text { Regressor }\end{array}$} & \multicolumn{3}{|c|}{ Reciprocity } & \multirow[t]{2}{*}{$\begin{array}{l}\text { Sense of } \\
\text { community }\end{array}$} & \multirow[t]{2}{*}{$\begin{array}{l}\text { Effective } \\
\text { Communicat } \\
\text { ion }\end{array}$} \\
\hline $\begin{array}{l}\text { Cultural } \\
\text { Belonging }\end{array}$ & $\begin{array}{c}0.436 \\
(0.178) \\
\end{array}$ & & & & & & & \\
\hline $\begin{array}{l}\text { Configuration of } \\
\text { SNS }\end{array}$ & & $\begin{array}{l}0.856 \\
(0.057)\end{array}$ & & & & & & \\
\hline Reciprocity & & & $\begin{array}{l}0.000^{* * *} \\
(0.046)\end{array}$ & & & & & \\
\hline $\begin{array}{l}\text { Configuration of } \\
\text { SNS }\end{array}$ & & & & $\begin{array}{c}0.730 \\
(0.075) \\
\end{array}$ & & & & \\
\hline $\begin{array}{l}\text { Native } \\
\text { Language }\end{array}$ & & & & & $\begin{array}{l}0.067^{* *} \\
(0.253)\end{array}$ & & & \\
\hline $\begin{array}{l}\text { Identification } \\
\text { Needs }\end{array}$ & & & & & & $\begin{array}{l}0.000^{* * * * *} \\
(0.055)\end{array}$ & & \\
\hline Trust & & & & & & & $\begin{array}{l}0.000^{* * * * *} \\
(0.053)\end{array}$ & \\
\hline $\begin{array}{l}\text { Sense of } \\
\text { Community }\end{array}$ & & & & & & & & $\begin{array}{l}0.000^{* * *+*} \\
(0.061)\end{array}$ \\
\hline $\mathrm{R}^{2}$ & & 0.460 & & & 0.375 & & 0.273 & 0.165 \\
\hline $\mathrm{F}$ & & 72.334 & & & 50.911 & & 96.570 & 50.669 \\
\hline $\mathrm{N}$ & & 259 & & & 259 & & 259 & 259 \\
\hline $\begin{array}{l}\text { Hypothesis } \\
\text { Supported }\end{array}$ & H1: No & H3: No & H6: Yes & H3: No & H2: No & H4: Yes & H5: Yes & H7: Yes \\
\hline
\end{tabular}

\section{Results}

\subsection{Hypothesis Testing}

We conducted multiple regressions to test our hypotheses using a level of significance of 0.05 . The results of hypotheses test are presented in Table 2.

The first regression was used to assess the relationship that trust, sense of community and cultural belonging have with effective communication. In the second regressions was used to examine the relationship that configuration of SNS and reciprocity have with trust in SNS interactions. We also regressed reciprocity in SNS interactions on native language variation, configuration of SNS, and identification needs. Finally, we regressed trust in SNS interactions on cultural belonging, configuration of SNS, and reciprocity of SNS.

The analyses supported hypothesis 4, 5,6 and 7. We found that identification needs had a significant effect on reciprocity in SNS interactions. In addition, we found support that trust in SNS interactions had a significant effect on sense of community We also found support for hypothesis 6 which suggests that reciprocity in SNS 
interactions is positively related to trust in SNS interactions. Finally, we found that the sense of community had a significant effect on effective communication. However, hypotheses 1, 2, 3, were not supported, as the researcher determined that cultural belonging has no significant effect on trust in SNS interactions and that native language has no significant effect on reciprocity in SNS interactions. In addition, the researcher found that configuration of SNS has no significant effect on trust or reciprocity in SNS interactions.

\section{Discussion}

The main goal of this study was to determine the effects of cultural belonging, native language variation, configuration of SNS, trust, reciprocity, sense of community and effective communication on the activities of social network sites. The study addressed the proposed hypothesis statements. The findings indicated that native language variation has no negative effect on reciprocity in SNS interactions, configuration of SNS has no effect on trust and reciprocity in SNS interactions. This finding deviates from literature indicating that SNS users typically set their SNS privacy settings favoring users that they trust or have reciprocal relationships with [35]. Privacy settings are a subset of configuration of SNS, this could possibly explain the deviation, and as such as the area of security and privacy with the configuration of SNS needs further research.

Further the findings indicated that identification needs have a positive effect on reciprocity in SNS interactions. This finding is consistent with literature, which suggested that psychological status of belonging to a community in an online social network can be stem from affective, evaluative and cognitive social identity [6]. In addition, the findings indicated that trust in SNS interactions has an effect on sense of community. These findings are consistent with prior studies that suggest that high levels of trust typically translate to people being more willing to provide support to other SNS members [18].

The findings also indicated that trust in SNS interactions increases with increased reciprocity in SNS interactions. This finding is consistent with literature that suggests that trust and reciprocity have a synergistic relationship, where reciprocity entails two users trusting each other in a two-way trust relationship. A network with numerous reciprocal linkages is likely to be more robust than one with fewer links of this nature [30]. The findings also indicated that sense of community has a positive effect on effective communication in SNS. These findings are consistent with prior research that suggests that where users feel a sense of community social interaction and effective communication is facilitated [29]. Finally, the findings indicated that cultural belonging had no statistically significant effect on trust in SNS interactions. These findings were somewhat consistent with prior research suggesting that, although new members of SNS typically seek out SNS familiar members with similar cultures or values, they eventually "friend' individuals with dissimilar cultures or values [10]. As SNS use proliferates globally, cultural belonging may no longer have an effect on trust in SNS interactions. The research in this study has implications for the information systems practice. The results of this study expound on the perspectives of the effects of trust in SNS interactions, reciprocity in SNS interactions and sense of community and effective communication on the activities of social network sites. This will enable the information systems field to appreciate how SNS users can communicate more effectively, once a level of trust, reciprocal collaboration and a sense of community is established on an SNS. This is essential for information systems field to understand, especially for developers as the tenets of SNS mirror the behavior traits of people in real life networks. Another implication for practice is related to how configuration of SNS, specifically configuration of security and privacy settings affect activities of social network sites. Security breaches and privacy violations of personal identifiable information (PII) are a current and prevalent topic in information technology. Consideration of SNS users' perception of security risks of sharing private and personal information is key in improving SNS use. Accordingly, analyzing the mechanisms necessary to incorporate better personal information sharing practices into the options available in configuration settings of a SNS is imperative for SNS developers and the information systems practice.

An implication for research concerns the identification of how configuration of SNS, specifically configuration of security and privacy settings could influence the activities of social network sites. Security breaches and privacy violations of personal identifiable information (PII) are prevalent, hence, this is a timely topic. While the results of this study did not support the hypothesis that stated that configuration of SNS has a positive effect on trust and reciprocity in SNS interactions, the finding deviates from literature indicating that SNS users typically set their SNS privacy settings favoring users that they trust or have reciprocal relationships with [35]. Further research will help the information system field to evaluate SNS users' perception of security risks of sharing their private and personal information and the effect of those perceptions on configuration of SNS. Accordingly, this study provides a basis for additional research necessary on various facets that affect interactions in SNS. 


\section{Limitations}

The first limitation in the study was that an automated survey was utilized to obtain responses from participants. Automated responses on SNS use that are self-reporting in nature may present certain limitations, as participants may be susceptible to providing socially acceptable answers. To alleviate this limitation, the researcher used a consent form with the participants, assuring the participants that their participation was voluntary and confidential. The second limitation was that the study was restricted to participants in the United States. Although the survey was only conducted in English language, approximately $10 \%$ of the participants that had an alternative native language and culture. Accordingly, in a globally based survey, the results may vary based on the geographic location of the participants.

\section{Conclusions and Future Research}

The study examined the factors that influence the development of the sense of community and effective communication in SNS interactions. This study expands upon prior studies on SNS interactions and recommends additional areas to consider in future research. The study found that identification needs, trust, reciprocity sense of community and effective communication all have an effect on SNS interactions. However, the study found that configuration of SNS did not have a significant effect on trust and reciprocity in SNS interactions, indicating that as the users' ability to configure SNS based on their preferences of security, privacy and other aspects evolving and need to be researched further. Accordingly, additional studies need to be performed to examine the effects of configuration of SNS on other constructs.

Considering the pervasive adoption and budding influence of SNS in the personal and professional lives of people globally, it is an emergent domain that has various opportunities for future studies. Research in the future ought to be conducted on a more global scale with participants drawn from a worldwide geographical area. In addition, future research could be conducted focusing on participants from specific age groups to determine the influence of SNS use at various life stages.

\section{References}

[1] Baker, W. and Dutton, J. E. "Enabling positive social capital in organizations," Exploring Positive Relationships at Work: Building a Theoretical And Research Foundation. 2007, pp. 325.
[2] Berg, J, Dickhaut, J and McCabe, K. "Trust, Reciprocity and Social History," Games and Economic Behavior, (10), 1995, pp. 122-142.

[3] Boyd, D. and Ellison, N. "Social Network Sites: Definition, History, and Scholarship," J. of Computer Mediated Communication, (13:1), 2007, pp. 210-230.

[4] Burke, M., Kraut, R and Marlow, C. "Social capital on Facebook: Differentiating uses and users," Proc. Ann. Conf. on human factors in computing systems, 2011, pp. 571-580.

[5] Burke M, Marlow C and Lento T. "Social network activity and social well-being," Proc. ACM Conf. on Human Factors in Computing Systems, 2010, 1909-1912.

[6] Cheung, C., Chiu, P. Y. and Lee, M. "Online social networks: Why do students use facebook?," Computers in Human Behavior, (27:4), 2011, pp. 1337-1343.

[7] Dwyer, C., Hiltz, S. R. and Passerini, K. "Trust and privacy concern within social networking sites: A comparison of Facebook and MySpace," Proc. of AMCI, 2007, pp. 339.

[8] Ellison, N. B., Steinfield, C. and Lampe, C. "Connection strategies: Social capital implications of Facebook-enabled communication practices," New Media \& Society, (13:6), 2011, pp. 873-892

[9] Gefen, D., Pavlou, P., Benbasat, I., McKnight, H., Stewart, $\mathrm{K}$ and Straub, D. "ICIS Panel Summary: Should Institutional Trust Matter in Information Systems Research?," Communications of the Association for Information Systems, (17), 2000, pp. 205-222.

[10] Gefen, D., Straub, D. W and Boudreau, M. C. "Structural equation modeling and regression: Guidelines for research practice. Communications of the Association for Information Systems," (4:7), 2013, pp. 1-77

[11] Grabner-Kräuter, S. and Bitter, S. "Trust in online social networks: A multifaceted perspective," In Forum for Social Economics," 2013, pp. 1-21.

[12] Gregor, S. "The nature of theory in information systems," MIS Quarterly, 2006, pp 611-642.

[13] Hofstede, G. In Culture's Consequences: International Differences in Work-Related Values, Sage., Newbury Park, CA, 1980.

[14] House, R., Javidan, M., Hanges, P. and Dorfman, P. "Understanding cultures and implicit leadership theories across the globe: an introduction to project GLOBE," J. of World Business (37), 2002, pp.3-10.

[15] Ji, Y., Hwangbo, H., Yi, J. S., Rau, P. P., Fang, X. and Ling, C. "The influence of cultural differences on the use of social network services and the formation of social capital," Intl. J. of Human-Computer Interaction (26:11), 2010. pp. 1100-1121.

[16] Kim, Y., Sohn, D and Choi, S. "Cultural differences in motivations for using social network sites: a comparative study of American and Korean college students," Computers in Human Behavior, (27:1), 2011, pp. 365-372.

[17] Kirkman, B., Lowe, K. B. and Gibson, C. "A quarter century of culture's consequences: A review of empirical research incorporating Hofstede's cultural values framework," J. of International Business Studies, (37:3), 2006, pp.285-320. [18] Krasnova, H., Spiekermann, S., Koroleva, K. and Hildebrand. "T. Online social networks: Why we disclose," J. of Information Technology, (25:2), 2010, pp.109-125.

[19] Lauterbach, D., Truong, H., Shah, T. and Adamic, L. Surfing a Web of Trust Reputation and Reciprocity on 
CouchSurfing. International Conference on Computational Science and Engineering. (2009).

[20] Lee, J. G., Antoniadis, P. and Salamatian, K. Faving Reciprocity in Content Sharing Communities: A Comparative Analysis of Flickr and Twitter. Proc. Int. Conf. on Advances in Social Networks Analysis and Mining (ASONAM), 2010.

[21] Leedy, P. D. and Ormrod, J. E. Practical research: Planning and design. Pearson., Upper Saddle River, NJ, 2012. [22] Lewicki, R. J. and Bunker, B. B. Developing and maintaining trust in work relationships. In R. M. Kramer \& T. M. Tyler (Eds.), Trust in organizations: Frontiers of theory and research, Sage., Thousand Oaks, CA, 1996.

[23] Lochner, K., Kawachi, I and Kennedy, B. "Social capital: a guide to its measurement," Health \& place, (5:4), 1999, pp. 259-270.

[24] Lowry, P., Zhang, Zhou, L and Xiaolan, F. "The Impact of National Culture and Social Presence on Trust and Communication Quality within Collaborative Groups," Proc. of the 40th Hawaii Int. Conf. on System Sciences, 2007, pp. 110 .

[25] Manvi, S. and Birje, M. N. "A review on wireless grid computing," International J. of Computer and Electrical Engineering, (2:3), 2010, pp. 1793-8163

[26] McMillan, D. W. and Chavis, D. M. "Sense of community: A definition and theory,". J. of community psychology, (14:1), 1986, pp. 6-23.

[27] Melitz, J. and Toubal, F. "Native language, spoken language, translation and trade," CEPR Discussion Paper (8994), 2012.

[28] Musembwa, S and Paul, S. "Social Networks: Cultural Diversity, Trust, Reciprocity and Social Capital," Proc. of AMCIS, 2012.

[29] Nahapiet, J. and Ghoshal, S. "Social capital, intellectual capital, and the organizational advantage. Academy of Management Review," (23:2), 1998, pp. 242-266.

[30] Nguyen, V., Lim, E., Tan, H., Jiang, J. and Suny, A. "Do You Trust to Get Trust? A Study of Trust Reciprocity Behaviors and Reciprocal Trust Prediction," Society of Industrial and Applied Mathematics (SIAM), 2010.
[31]; Portes, A. 1998. "Social capital: Its origins and applications in modern sociology, “. Annual Review of Sociology, (24), 1998, pp. 1-24

[32] Robbins, S. S and Stylianous, A. "A longitudinal study of cultural differences in global corporate web sites," J. of International Business and Cultural Studies, (3), 2010, pp. 7796.

[33] Rumbaut, R. and Massey, D. "Immigration \& Language Diversity in the United States. Daedalus," (142:3), 2013. pp. 141-154.

[34] Sekaran, U. and Bougie, J. Research Methods for Business: A Skill Building Approach (7th edition), John Wiley and Sons, Inc., Hoboken, NJ. 2016.

[35] Sledgianowski, D. and Kulviwat, S. "Using Social network sites the effect of playfulness critical mass and trust in a hedonic context," The J. of Computer Information Systems, (49:4), 2009. pp. 74-83.

[36]; Steinfield, C., Ellison, N. and Lampe, C. "Social Capital, Self-esteem, and Use of Online Social Network Sites: A longitudinal analysis," J. of Applied Developmental Psychology, (29), 2008, pp. 434-445.

[37] Tajfel, H. Social categorization, social identity, and social comparison. In Differentiation between social groups: Studies in the social psychology of intergroup relations. Academic Press., London, England, 1978.

[38] Tang, L. and Koveos, P. E. "A framework to update Hofstede's cultural value indices: economic dynamics and institutional stability," J. of International Business Studies, (39:6), 2008, pp, 1045-1063.

[39] Backstrom L, Huttenlocher D, Kleinberg J, and Lan X. Group formation in large social networks: membership, growth, and evolution. InProc. of the 12th ACM SIGKDD Int. Conf. on Knowledge discovery and data mining 2006 Aug 20 pp. 44-54. ACM.

[40] Wasko MM and Faraj S. Why should I share? Examining social capital and knowledge contribution in electronic networks of practice. MIS quarterly. (1;29) 2005 Mar pp, 3557. 